\title{
部说 CEREUS
}

\section{AVALIAÇÃO DO USO E CONHECIMENTO DE SUPLEMENTOS ALIMENTARES POR ACADÊMICOS DO CURSO DE EDUCAÇÃO FISICA}

\section{OLIVEIRA, Susane Rayanne U. de ${ }^{1}$ MARIN, Kliver Antonio ${ }^{2}$ PEREIRA, Renata Junqueira ${ }^{3}$. RESUMO NASCIMENTO, Guilherme Nobre L. do ${ }^{4}$}

O padrão de consumo de suplementos alimentares, entre outros fatores, ocorre a partir da orientação de instrutores ou professores de educação física com a finalidade estética elou de aumentar as metas da massa muscular. Com este estudo foi avaliado o uso e conhecimento de suplementos alimentares em uma amostra de estudantes de graduação de Educação Física. Foi realizado uma entrevista semiestruturada em três etapas: a primeira foi relativa a aspectos socioculturais, na segunda foram feitas perguntas

\footnotetext{
${ }^{1}$ Farmacêutica, Mestre em Ciências da Saúde - Universidade Federal do Tocantins (UFT).

${ }^{2}$ Fisioterapeuta, Doutorando em Ciências da Nutrição - UFV. Professor do curso de Educação Física - UFT.

${ }^{3}$ Nutricionista, Doutora em Ciência de Alimentos. Professora dos cursos de Nutrição, Mestrado em Ciências da Saúde e Mestrado em Ciência e Tecnologia de Alimentos UFT.

${ }^{4}$ Farmacêutico. Doutor em Química. Professor dos cursos de Nutrição, Mestrado em Ciências da Saúde e Mestrado em Ciência e Tecnologia de Alimentos. Laboratório de Ciências Básicas e da Saúde (LaCiBS) - UFT. guilherme.nobre@uft.edu.br
}

Rev. Cereus, v. 9, n. 3, p.35-48, set-dez./2017, UnirG, Gurupi, TO, E

v. 9, n. 3, set/dez. 2017 UnirG, Gurupi, TO, Brasil 
relacionadas à orientação e conhecimento sobre suplementos alimentares, na terceira foi aplicado um teste para relacionar o tipo de suplemento com a sua respectiva função. Foram entrevistados 224 alunos de ambos os sexos no curso de graduação em educação física, sendo, $45,1 \%$ feminino e $54,9 \%$ masculino. Destes, $19,6 \%$ relataram uso de suplementos alimentares, e destes a média de acerto das questões da terceira etapa da entrevista foi de $58,7 \%$. Os resultados sugerem um baixo conhecimento sobre suplementos alimentares, havendo assim necessidade de mais conteúdos sobre nutrição esportiva na formação dos educadores físicos.

Palavras chave: Suplementos nutricionais, educação física, nutrição esportiva.

\section{EVALUATION OF USE AND KNOWLEDGE OF DIETARY SUPPLEMENTS IN PHYSICAL EDUCATION STUDENTS}

\section{ABSTRACT}

The pattern of consumption of dietary supplements, among other factors, occurs from the orientation of instructors or teachers of physical education with the aesthetic purpose and/or goals to increase the muscle mass. This study evaluated the use and knowledge of dietary supplements in a sample of undergraduate students of Physical Education. A semistructured interview was carried out in three stages: the first one was related to socio-cultural aspects; the second one asked questions related to orientation and knowledge about dietary supplements; in the third, a test was applied to relate the type of supplement to its respective function. We interviewed 224 students of both genders in the undergraduate course in physical education, being $45.1 \%$ female and $54.9 \%$ male. Of these, $19.6 \%$ reported use of dietary supplements, and of these the average accuracy of 
questions in the third stage of the interview was $58.7 \%$. The results suggest a low knowledge about dietary supplements, thus requiring more content on sports nutrition in the training of physical educators.

Keywords: Dietary Supplements, physical education, sport nutrition 


\section{INTRODUÇÃO}

O benefício de uma correta alimentação há tempos é reconhecido e Órgãos Nacionais e Internacionais tem criado diretrizes e guias alimentares e dietéticos para orientar o correto consumo dos alimentos e seus nutrientes (TACO, 2011; USDHHS/USDA, 2015; SBMEE, 2009). Porém observa-se 0 crescente consumo de substâncias isoladas, manufaturadas, extratos entre outros produtos administrados para suplementação dietética (CHIBA et tal., 2014) principalmente entre os praticantes de atividades físicas (GALATI et al 2017; FRINHANI e LEONHARDT 2016). Tais produtos recebem o nome de suplementos alimentares (SA). Mas seu uso tem que ser corretamente indicado e orientado uma vez que o aumento da concentração das substâncias em uma dose ou alimento, aumenta a probabilidade de ocorrência de efeitos adversos ou até mesmo tóxico (ADA, 2005).

De acordo com alguns autores (CORRÊA e NAVARRO, 2014; GOSTON e CORREIRA, 2010; LACERDA et al., 2015), o termo SA refere-se a quaisquer produtos feitos à base de vitaminas, minerais, plantas medicinais, proteínas, aminoácidos, metabólitos (creatina e carnitina) e produtos utilizados para a melhoria de desempenho e composição corporal. Porém, esta definição pode sofrer variações de acordo com os autores ou mesmo órgãos de regulação.

Estudos vem sendo realizado em diferentes cidades do Brasil (ARAÚJO et al., 2002; DOMINGUES e MARIN 2007; SANTOS e SANTOS,2002; PEREIRA et al., 2003; LOPES et al., 2015; LINHARES e LIMA, 2006; HIRSCHBRUCH et al., 2008; GOSTON e CORREIA, 2010) que observaram como objetivos para esta suplementação o caráter estético e aumento de massa muscular na maioria dos casos, sendo indicados por instrutores, treinadores pessoais e educadores físicos em academias. Logo, os profissionais da área da saúde para auxiliar a população quanto ao adequado uso destes recursos, dependem de uma formação acadêmica adequada e isto pode ser verificado logo do curso de graduação. Portanto, o objetivo deste estudo foi avaliar o consumo e o conhecimento sobre SA em uma amostra de 
estudantes de educação física.

\section{MATERIAIS E MÉTODOS}

Foi realizado um estudo transversal descritivo, através de entrevista semi-estruturada, com estudantes de educação física, de ambos os sexos, matriculados em um curso de bacharelado, presencial, noturno de faculdade particular de Goiânia, Brasil. Foram incluídos no estudo, alunos devidamente matriculados do $1^{\circ}$ ao $8^{\circ}$ período do curso de Educação Física com idade igual ou superior a 18 anos. Participaram da pesquisa 224 indivíduos, 28 de cada período, abordados em diferentes horários e dias da semana, em demanda espontânea, nos corredores da faculdade. Após serem esclarecidos a respeito do objetivo, procedimentos e riscos desta pesquisa, os mesmos foram convidados a assinar um termo de consentimento livre e esclarecido, de acordo com a resolução específica do Conselho Nacional de Saúde (no 466/12). Este trabalho foi aprovado pelo Comité de Ética em Pesquisa da Universidade Estácio de Sá, sob protocolo: 527.753 de 2014.
Para a entrevista foi utilizado um questionário, previamente elaborado baseado na literatura pertinente (ROCHA e PEREIRA, 1998; STEYN et al., 2005; NASCIMENTO et al., 2013) e testado em um estudo piloto com 30 participantes para correções de possíveis problemas da metodologia e no instrumento. Através do teste piloto algumas perguntas foram suprimidas e outras foram reescritas para melhor entendimento dos participantes. O questionário foi composto por três partes: a primeira diz respeito a questões socioculturais, a segunda sobre questões referentes ao uso de suplementos alimentares e a terceira parte foi referente ao conhecimento dos estudantes sobre SA, com questões diretas relacionando os diferentes tipos de suplementos e suas indicações. O objetivo destas questões foi verificar o conhecimento dos estudantes, comparando a evolução dos mesmos entre os períodos e o percentual de acertos e erros.

Os diferentes tipos de SA foram agrupados em: i) suplementos ricos em proteínas e aminoácidos; ii) vitaminas, 
multivitaminas / minerais; iii) aqueles ricos em carboidratos; iv) a creatina ; v) I- carnitina ; vi) isotônicos; e vii) naturais / fitoterápicos.

Os dados foram agrupados e analisados por meio de estatística

\section{RESULTADOS}

Foram entrevistados 224 alunos do primeiro ao oitavo período do curso de Educação Física, sendo $45,1 \%$ do sexo feminino e $54,9 \%$ do masculino. Dos entrevistados $4,9 \%$ tinham idade entre 18 e 20 anos, 86,6\% tinham entre 21 e 30 anos, $8,5 \%$ entre 31 e 40 anos. descritiva, teste de análise de variância e teste de Tukey ao nível de significância de $5 \%$, utilizando para estas análises o software Graphpad Prism 6.0

Quanto à renda mensal dos indivíduos, $91,5 \%$ relataram entre um e quatro salários mínimos, 1,8\% ganham menos de um salário mínimo e 6,7\% ganham acima de quatro salários mínimos (Tabela 1).

Tabela 1. Frequência de respostas relacionadas ao gênero, idade e renda mensal.

\begin{tabular}{llll}
\hline & & FA (n) & FR (\%) \\
\hline Gênero & Feminino & 101 & 45 \\
& Masculino & 123 & 55 \\
\hline & $\leq 20$ anos & 11 & 5 \\
& $21-30$ & 194 & 87 \\
& $31-40$ & 19 & 8 \\
& $\geq 40$ & 0 & 0 \\
\hline Renda Mensal & $<1$ salário mínimo & 4 & 1,8 \\
& $1-4$ salários minímos & 205 & 91,5 \\
& $>$ de 4 salários mínimos & 15 & 6,7 \\
\hline
\end{tabular}

$*_{n}=\overline{224}$

Para a pergunta: "você pratica atividade física", 99,6\% responderam que sim, destes indivíduos, a maioria relatou como motivação para a prática da atividade física o "bem estar físico e mental", 22\% "manutenção de peso", e $15,7 \%$ "ganho de massa muscular" (Figura. 1). A atividade física mais praticada foi à musculação com $41,9 \%$ (Figura. 2). 
Figura 1. Frequências de respostas para as perguntas sobre os objetivos que motiva os indivíduos para a prática da atividade física $(\mathrm{n}=223)$.

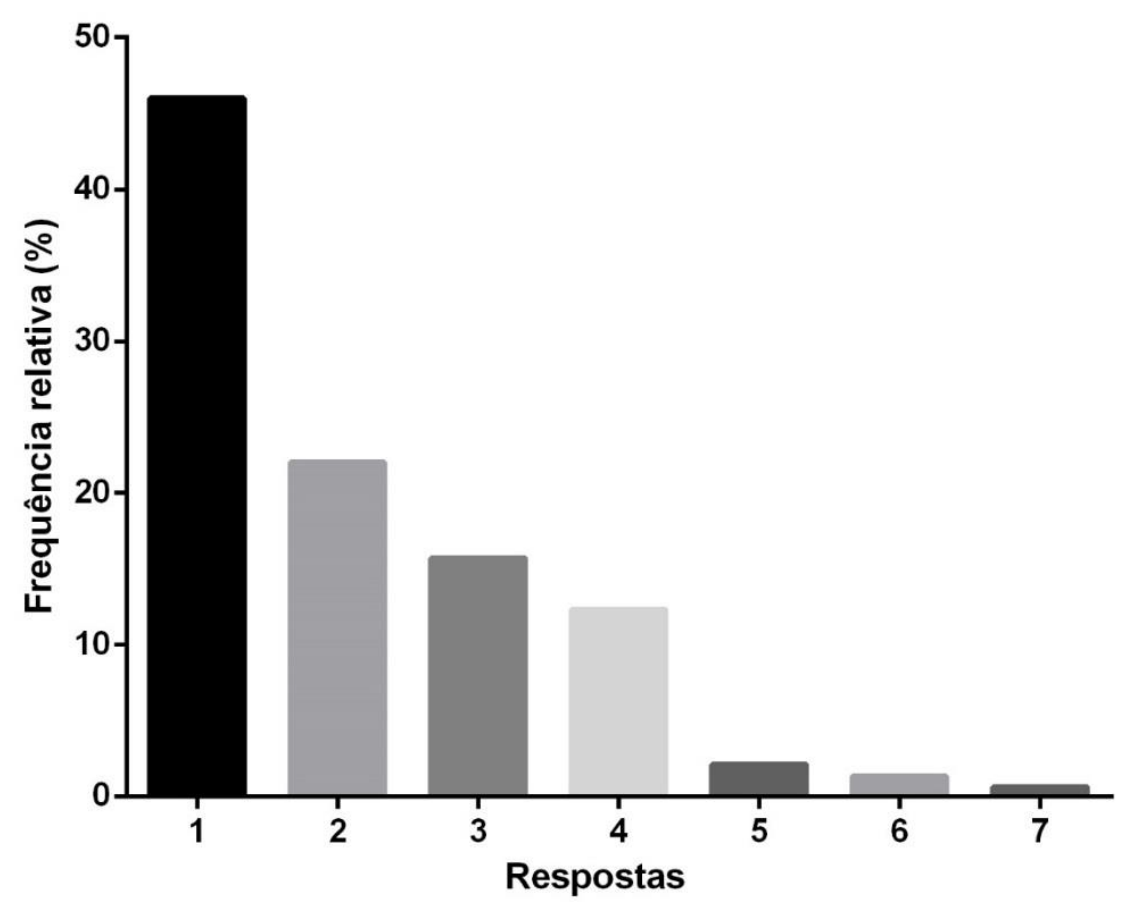

Respostas: 1-Bem estar físico e mental; 2-Manutenção do peso; 3- Ganho de massa muscular; 4Estética; 5- Perda de peso; 6- Outro; 7- Recomendação médica.

Figura 2. Frequências de respostas para as perguntas sobre qual atividade física o indivíduo pratica $(\mathrm{n}=223)$.

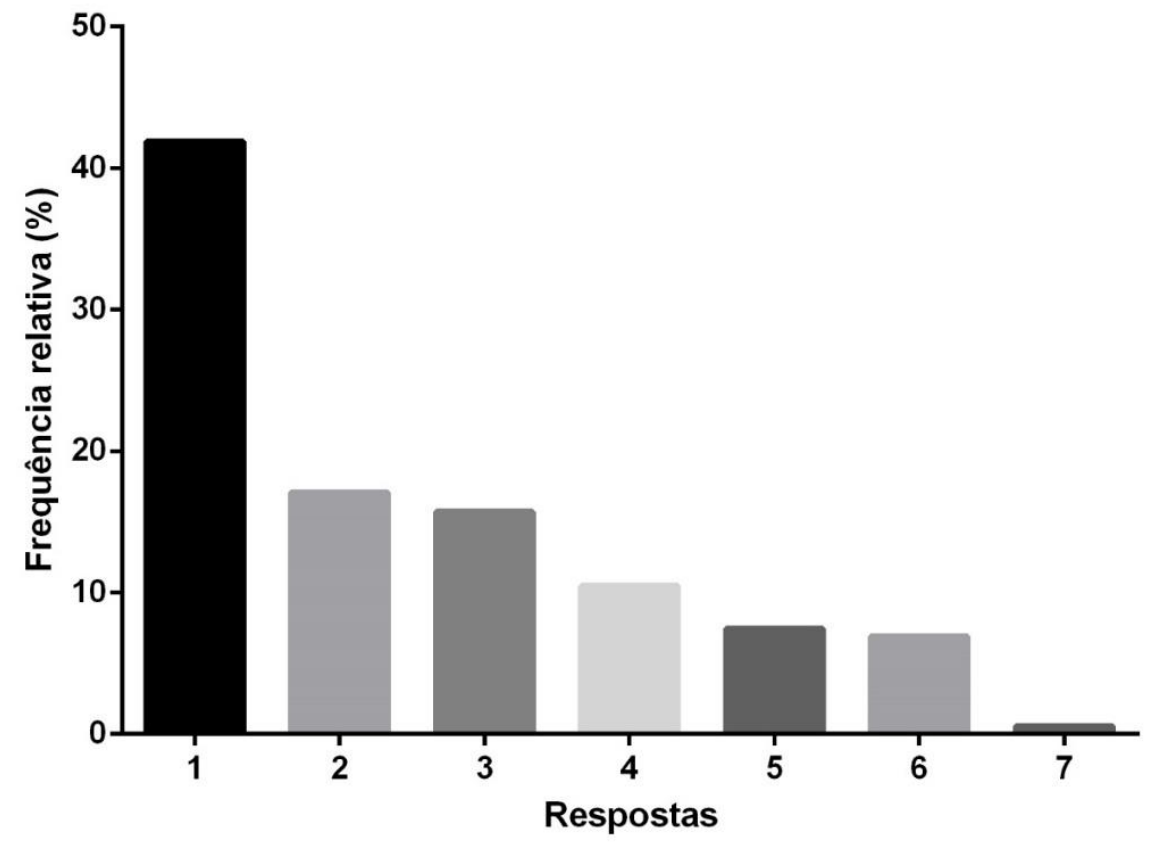

Respostas: 1-Musculação; 2-Caminhada; 3-Luta; 4-Corrida; 5-Natação; 6-Ciclismo; 7-Outra. Rev. Cereus, v. 9, n. 3, p.35-48, set-dez./2017, UnirG, Gurupi, TO, Brasil. 
Sobre a pergunta "Você utiliza algum suplemento alimentar? ", 19,6\% relataram uso de suplementos alimentares. Dentre os usuários, 100\% relataram nunca ter apresentado qualquer efeito colateral, apesar de todos terem informado conhecer que os mesmos podem causar efeitos indesejados. Os suplementos mais consumidos foram bebidas isotônicas $(33,3 \%)$ e aminoácidos (25,4\%) (Figura. 3).

Figura 3. Frequência de respostas para as perguntas sobre qual tipo de suplemento o indivíduo consome $(n=44)$.

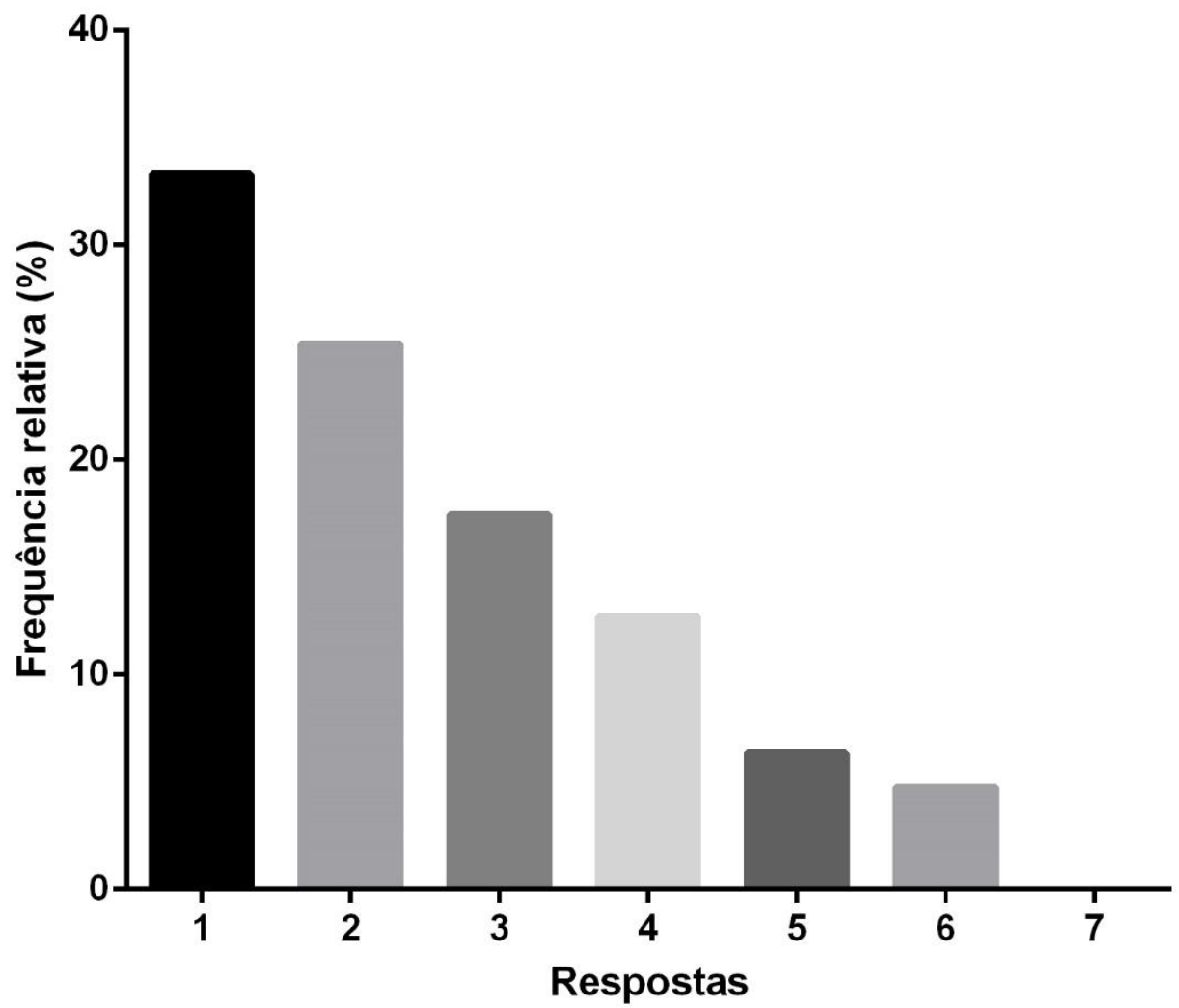

Respostas: 1- Bebidas isotônicas; 2- Aminoácidos ou outros concentrados proteicos; 3- Vitaminas ou complexos vitamínicos; 4- Creatina; 5- Produtos fitoterápicos; 6- L-Carnitina; 7-Outra.

Foi aplicado um teste de associação para os estudantes entre 0 nome do suplemento alimentar e a sua função correspondente. A média de respostas corretas dos indivíduos foi de $58,7 \%$. Os índices de acertos entre o primeiro e o oitavo período sofreram variações, porém mais acentuadas apenas no oitavo período (Figura. 4). Para cada grupo de suplementos, o que apresentou a maioria dos acertos foi 0 de produtos fitoterápicos (15,5\%), as vitaminas e bebidas isotônicas (15,4\%) (Figura 5). 
Figura 4. Média dos acertos na prova de conhecimento sobre suplementos por período do curso $(\mathrm{n}=224)$.

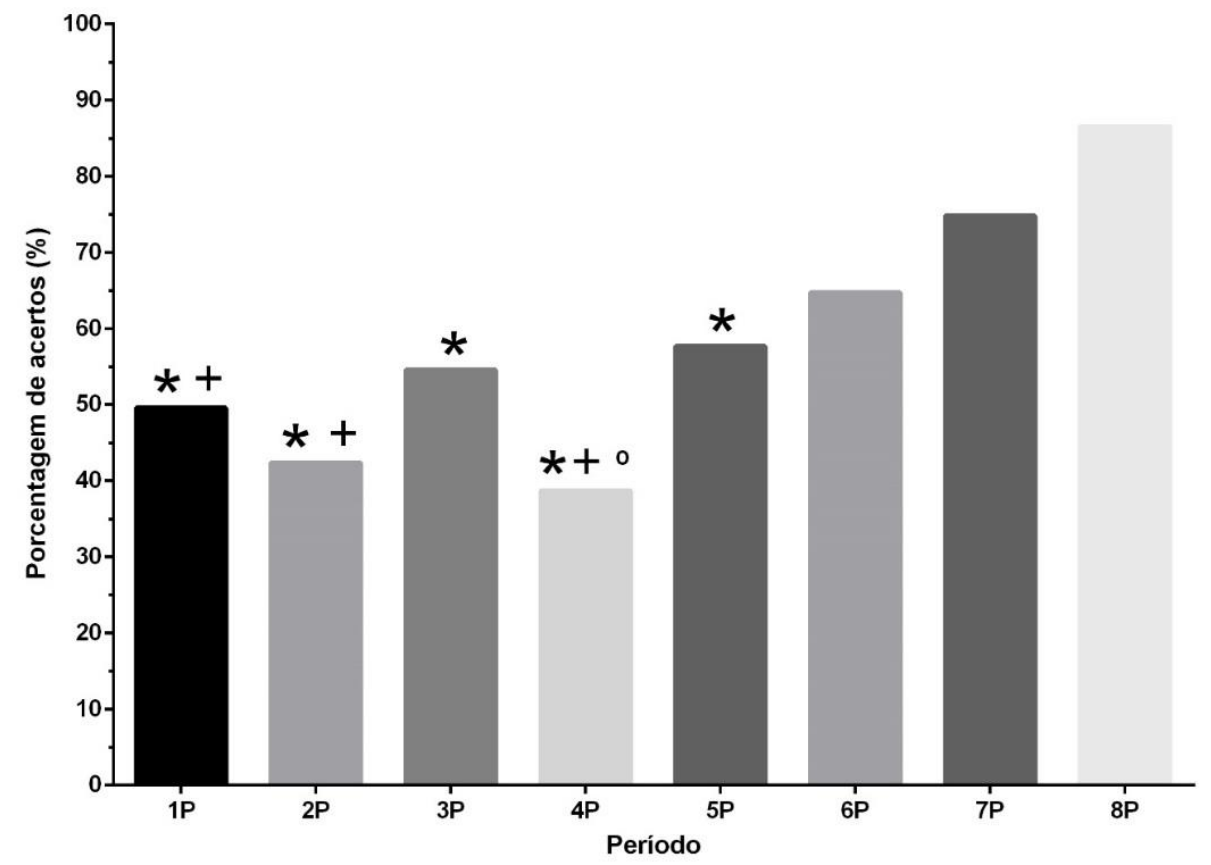

*Diferente significativamente em comparação ao $8^{\circ}$ período (8P) para $p<0.05$; +Diferente significativamente em comparação ao $7^{\circ}$ período (7P) para $p<0.05$; o Diferente significativamente em comparação ao $6^{\circ}$ período (6P) para $p<0.05$.

Figura 5. Porcentagem dos acertos na prova de conhecimento sobre suplementos por questão referente a cada suplemento $(n=224)$.

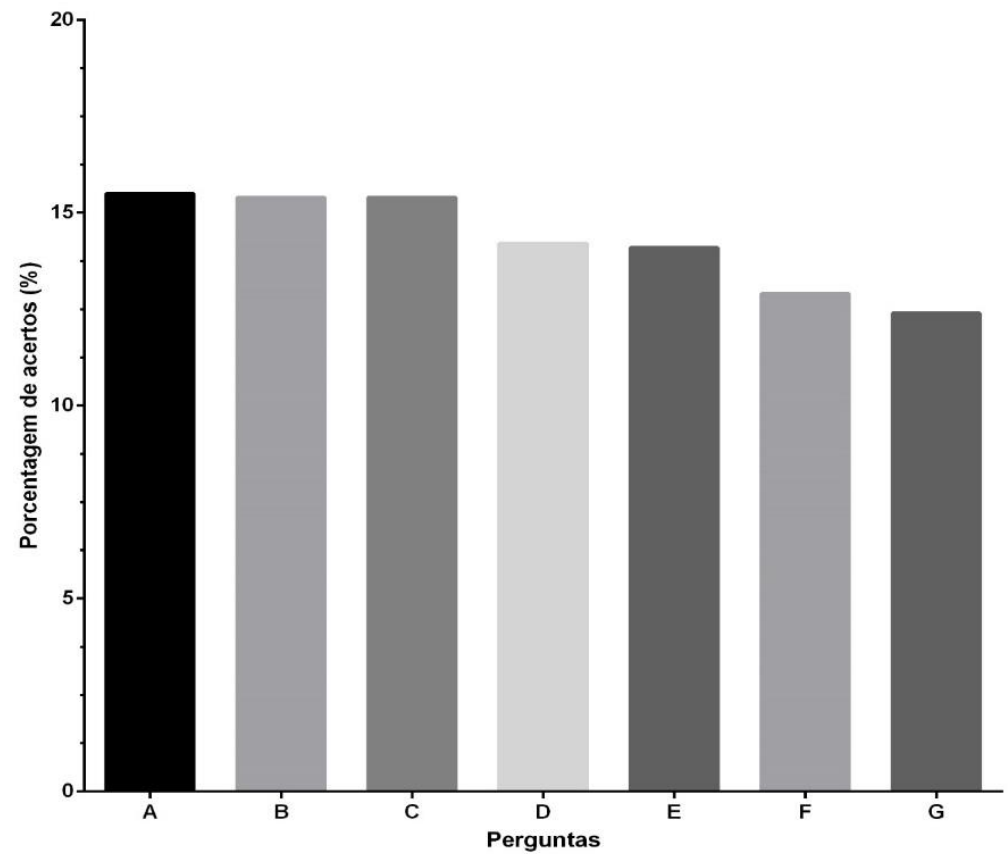

A-Produtos Fitoterápicos; B-Vitaminas; C-Bebidas Isotônicas; D-Carnitina; E-Carboidratos; FCreatina; G-Aminoácidos.

Rev. Cereus, v. 9, n. 3, p.35-48, set-dez./2017, UnirG, Gurupi, TO, Brasil. 


\section{DISCUSSÃO}

Quase a totalidade dos entrevistados praticam atividade física $(99,6 \%)$ sendo a musculação a atividade mais reportada $(41,9 \% \%)$. Os dados demonstram que as razões para tal escolha estão relacionadas aos interesses de manutenção da saúde e do corpo, diferente da observada em estudos realizados em academias em que os entrevistados citaram como o principal interesse para a prática de musculação, o ganho de massa muscular (JOST e POLL, 2014; FAYH et al., 2013; GOSTON e CORREIA, 2010; ARAÚJO et al., 2002; TIAN et al., 2009; PALMER et al., 2003). Esta divergência pode ser devido ao fato de que os alunos têm contato com os conceitos de saúde e a opção por um curso de educação física está associada a pessoas que gostam de exercitar e manterem-se saudáveis, e a população leiga pode associar ganho de massa muscular à boa forma estética e à aceitação social (HIRSCHBRUCH et al., 2008; VIEIRA et al., 2010). Esta é uma questão complexa e é um comportamento que requer mais pesquisas (ARAÚJO et al., 2002; PALMER et al., 2003).
Por outro lado, os suplementos mais citados quanto ao consumo foram às bebidas isotônicas e os complexos de aminoácidos e proteicos, o que corrobora $O$ encontrado em outros estudos (PEREIRA et al., 2003; ROCHA e PEREIRA, 1998; MIARKA et al., 2007; O'DEA, 2003). Com isto, pode-se inferir, que mesmo tendo respondido como principal objetivo para a prática da atividade física o bem-estar físico e mental, o perfil de uso de suplementos aponta para o consumo de substâncias da moda nas academias.

Todos os entrevistados citaram conhecer que os suplementos alimentares podem causar efeitos colaterais, porém nenhum citou já ter apresentado algum destes efeitos. Podemos aqui hipotetizar que os alunos podem realmente não conhecer quais são os sinais e sintomas de efeitos colaterais ou por outro lado que realmente não foram observados, uma vez que estes podem demorar para ocorrer após o uso, ou até mesmo não serem interpretados como provenientes do consumo de suplementos, como é o caso do aparecimento de espinhas e aumento da oleosidade da pele. 
Os problemas associados com o uso incorreto podem causar hemorragia cerebral, tonturas, dor no peito, arritmia, bradicardia, infarto do miocárdio, hepatotoxicidade, dispnéia, depressão respiratória e até mesmo coma (PALMER et al., 2003). Logo apesar de indicar um uso com foco na saúde, o uso dos suplementos indica uma finalidade estética e muito próxima ao uso realizado da população em geral.

$\mathrm{Na}$ prova de conhecimento quanto a função de cada uma das classes de suplementos, foi observado baixos níveis de acertos até o sexto período, tendo uma melhora no sétimo período, porém este não se difere significativamente do 3 e do 5 período, e o oitavo período com diferença significativa em relação aos primeiros períodos do curso. Vale ressaltar aqui que as perguntas eram simples, e referentes unicamente a função básica de cada um dos suplementos. O curso tem duração de 4 anos de duração, realizado no período noturno e não apresenta disciplinas específicas voltadas a essa temática, apenas os conteúdos de Bioquímica e Fisiologia onde foi verificado que o professor abordava esporadicamente este assunto. Quanto à porcentagem de respostas em cada um dos tipos de suplementos, aquele com menor nível de acertos foi para os aminoácidos e compostos proteicos (Fig. 5), segunda classe mais consumida pelos participantes deste trabalho.

Apenar de boa pontuação dos alunos dos dois últimos períodos do curso, foram identificadas preocupações referente a esta temática como os efeitos colaterais e finalidade de uso destas substâncias. Mesmo não sendo os profissionais que irão trabalhar diretamente na prescrição destas substâncias, mas irão trabalhar com clientes em academias e que cada vez mais buscam na suplementação uma alternativa para seu ganho de massa muscular, perda de peço e para fins estéticos, e que tem no educador físico um profissional de referência obre a prática da atividade física. Sendo assim necessário estudos mais aprofundados sobre a base curricular deste curso e estudos com questões mais específicas em relação aos processos bioquímicos envolvidos no uso destes suplementos.

\section{CONCLUSÃO}


4. Podemos concluir com este trabalho que o consumo de suplementos é elevado e semelhante ao de praticantes de atividade física em academias. $\mathrm{O}$ nos últimos dois períodos demonstrou questões a serem avaliadas quanto ao conhecimento de efeitos colaterais e finalidades de uso. conhecimento apesar de bom

\section{AGRADECIMENTOS}

Os autores agradecem a Universidade Federal do Tocantins pelas bolsas concedidas.

\section{REFERÊNCIAS}

ADA - American Dietetic Association. Position of the American Dietetic Association: fortification and nutritional supplements. Journal of the American Dietetic Association. v.105, n. 8, p. 1300-11, 2005.

ARAÚJO, L. R.; ANDREOLO, J.; SILVA, M. S. Utilização de suplemento alimentar e anabolizante por praticantes de musculação nas academias de Goiânia-GO. Revista Brasileira de Ciência e Movimento. v. 10, p. 13-18, 2002.

CHIBA, T.; SATO, Y.; NAKANISHI, T.; YOKOTANI, K.; SUZUKI, S.; UMEGAKI, K. Inappropriate usage of dietary supplements in patients by miscommunication with physicians in Japan. Nutrients. n. 6, p. 5392-5404, 2014.

CONSELHO NACIONAL DE SAÚDE. Diretrizes e normas regulamentadoras de pesquisas envolvendo seres humanos. Resolução 466 de 10 de outubro de 2012, 2012.

CORRÊA, D. B.; NAVARRO, A. C. Distribuição de respostas dos praticantes de atividade física com relação à utilização de suplementos alimentares e o acompanhamento nutricional numa academia de Natal/RN. Revista Brasileira de Nutrição Esportiva. v. 8, n. 43, p.35-51, 2014.

DOMINGUES, S. F.; MARINS, J. C. B. Utilização de recursos ergogênicos e suplementos alimentares por praticantes de musculação em Belo Horizonte - MG. Fitness and Performance Journal. v. 6, n. 4, p. 218-226, 2007.

FAYH, A. P. T.; SILVA, C. V.; JESUS, F. R. D.; COSTA, G. K. Consumo de suplementos nutricionais por frequentadores de academias da cidade de Porto Alegre. Revista Brasileira de Ciências do Esporte. v. 35, p. 27-37, 2013. 
FRINHANI, T. O.; LEONHARDT, V. Consumo de suplementos alimentares enriquecidos de vitaminas e minerais, e/ou suplemento vitamínico-mineral em uma universidade privada de Brasília-DF. Revista Brasileira de Nutrição Esportiva. v. 10, n. 60, p.654-659, 2016.

GALATI, P. C.; GIANTAGLIA, A. P. F.; TOLEDO, G. C. G. Caracterização do consumo de suplementos nutricionais e de macronutrientes em praticantes de atividade física em academias de Ribeirão Preto-SP. Revista Brasileira de Nutrição Esportiva. v. 11, n. 62, p.150-159, 2017.

GOSTON, J. L.; CORREIA, M. I. T. D. Intake of nutritional supplements among people exercising in gyms and influencing factors. Nutrition, v. 26, p. 604-611, p. 2010.

HIRSCHBRUCH, M. D.; FISBERG, M.; MOCHIZUK, L. Consumo de Suplementos por Jovens Freqüentadores de Academias de Ginástica em São Paulo. Revista Brasileira de Medicina do Esporte. v. 14, p. 539- 543, 2008.

JOST, P. A.; POLL, F. A. Food supplements intake among practitioners of physical activity in gyms at Santa Cruz do Sul - RS. Cinergis. v. 15, p. 10-17, 2014.

LACERDA, F. M. M.; CARVALHO, W. R. G.; HORTEGAL, E. V.; CABRAL, N. A. L.; VELOSO, H. J. F. Factors associated with dietary supplement use by people who exercise at gyms. Revista de Saúde Pública. v.49, 63, p. 2015.

LINHARES, T. C.; LIMA, R. M. Prevalência do uso de suplementos alimentares por praticantes de musculação nas academias de Campos dos Goytacazes/RJ, Brasil. Vértices. v. 8, n. 1/3, p. 101-122, 2006.

LOPES, F. G.; MENDES, L. L.; BINOTI, M. L.; OLIVEIRA, N. P.; PERCEGONI, N. Conhecimento sobre nutrição e consumo de suplementos em academias de ginástica de Juiz de Fora, Brasil. Revista Brasileira de Medicina do Esporte. v. 21, n. 6, 2015.

MIARKA, B.; JUNIOR, C. C. L.; INTERDONATO, G. C.; DEL VECCHIO, F. B. Características da suplementação alimentar por amostra representativa de acadêmicos da área de educação física. Revista Movimento e Percepção. v. 8, p. 278-288, 2007.

NASCIMENTO, M. V. S.; RAPOSO, O. F. F.; BRITO NETTO, R. S. M. Conhecimento em nutrição de instrutores de musculação do município de Aracaju - SE. Revista Brasileira de Ciências do Esporte. v. 35, n. 4, p. 1051-1070, 2013.

O'DEA, J. A. Consumption of nutritional supplements among adolescents: usage and perceived benefits. Health education research: theory and practice. v. 18, p. 98107, 2003.

PALMER, M. E.; HALLER, C.; MCKINNEY, P. E.; KLEIN-SCHWARTZ, W.; TSCHIRGI, A.; SMOLINSKE, S. C. Adverse events associated with dietary supplements: an observational study. Lancet. v. 361, p. 101-106, 2003. 
PEREIRA, R.F.; LAJOLO, F. M.; HIRSCHBRUCH, M. D. Consumo de suplementos por alunos de academias de ginástica em São Paulo. Revista de Nutrição. v. 16, p. 265-272, 2003.

ROCHA, L.P.; PEREIRA, M. V. L. Consumo de suplementos nutricionais por praticantes de exercícios físicos em academias. Revista de Nutrição. v. 11, p. 76-82, 1998.

SANTOS, M. A. A.; SANTOS, R. P. Uso de suplementos alimentares como forma de melhorar a performance nos programas de atividade física em academias de ginástica. Revista Paulista de Educação Física. v. 16, p. 174-185, 2002.

SBMEE - Sociedade Brasileira de Medicina do Exercício e do Esporte. Modificações dietéticas, reposição hídrica, suplementos alimentares e drogas: comprovação de ação ergogênica e potenciais riscos para a saúde. Revista Brasileira de Medicina do Esporte. v. 15, n. 3, supl. p. 2-12, 2009.

STEYN, N. P.; DEMETRE LABADARIOS, M. B; JOHANNA, H. N.; HEIDI-LEE, R. Development and validation of a questionnaire to test knowledge and practices of dietitians regarding dietary supplements. Nutrition. v. 21, p. 51-58, 2005.

TACO - Tabela brasileira de composição de alimentos. NEPA/UNICAMP. 4. ed. rev. e ampl. Campinas, 2011. $161 \mathrm{p}$.

TIAN, H. H.; ONG, W. S.; TAN, C. L. Nutritional supplement use among university athletes in Singapore. Singapore Medical Journal. v. 50, p. 165-172, 2009.

USDHHS/USDA - U.S. Department of Health and Human Services and U.S. Department of Agriculture. 2015 - 2020 Dietary Guidelines for Americans. 8th Edition. December 2015. Available at http://health.gov/dietaryguidelines/2015/guidelines/.

VIEIRA, J. L. L.; ROCHA, P. G. M.; FERRAREZZI, R. A. A dependência pela prática de exercícios físicos e o uso de recursos ergogênicos. Acta Scientiarum - Health Sciences. v. 32, p. 35-41, 2010.

Recebido em: 08/09/2017

Aprovado em: 08/11/2017 